\title{
New Cosmology: The Global Dynamics of the Higgs Quantum Space and the Accelerated Expansion of the Universe
}

\author{
Jacob Schaf \\ Universidade Federal do Rio Grande do Sul (UFRGS), Instituto de Fsica, Porto Alegre-RS, Brazil \\ Email: schaf@if.ufrgs.br
}

How to cite this paper: Schaf, J. (2019) New Cosmology: The Global Dynamics of the Higgs Quantum Space and the Accelerated Expansion of the Universe. Journal of Modern Physics, 10, 281-293.

https://doi.org/10.4236/jmp.2019.103019

Received: November 7, 2018

Accepted: March 4, 2019

Published: March 7, 2019

Copyright $\odot 2019$ by author(s) and Scientific Research Publishing Inc. This work is licensed under the Creative Commons Attribution International License (CC BY 4.0).

http://creativecommons.org/licenses/by/4.0/

\begin{abstract}
This work investigates the nature of the empty space and of the energy accelerating expansion of the universe, within the context of the Higgs theory. It is consensus among the cosmologists that dark energy, accelerating the expansion of the universe, is energy of the empty space (vacuum) itself. According to the Higgs theory, empty space (vacuum) is filled up by a real quantum fluid medium, closely analogous to the superconducting condensate, giving mass to the elementary particles by the Higgs mechanism. This spatial medium is the holder of the vacuum energy. Current theories describe the empty space (vacuum) in terms of the stress-energy tensor of a perfect fluid and estimate the vacuum energy density in terms of zero-point energies of the various force fields. They come to the scandalous conclusion that the vacuum energy density is 120 decimal orders of magnitude larger than shown by the observations. In the context of the Higgs theory, empty space, far from a perfect fluid, is a very strongly correlated boson condensate, a perfect quantum fluid ruled by the principles of quantum physics and governed by a powerful order parameter. This order parameter is stabilized by a huge energy gap that, according to the Glashow-Weinberg-Salam electroweak model, achieves more than $200 \mathrm{GeV}$. This huge energy gap very strongly suppresses the quantum fluctuations and the zero-point energies. This lets clear that estimating the vacuum energy density in terms of the zero-point energies cannot be correct. The expanding universe does not create more and more vacuum energy and does not expand against a negative pressure. The universe is an adiabatic system that conserves the total mass-energy and expansion only reduces the vacuum energy density. Calculations within this context show that the vacuum energy density converges closely to the observed value.
\end{abstract}

\section{Keywords}

Dark Energy, Vacuum Energy, Cosmological Constant, Higgs Theory, Expanding 


\section{Introduction}

When Friedman [1] discovered that the solution of Einstein's field equations [2] [3] of General Relativity (GR) in a Robertson-Walker universe, [4] [5] leads to an expanding universe, Einstein immediately has included a cosmological term to get solutions for a static universe, because, in his view, only a static universe could be reasonable. The field equations, including the cosmological term took the form:

$$
R_{\mu \nu}-\frac{1}{2} g_{\mu v} R+\Lambda g_{\mu v}=8 \pi G T_{\mu v},
$$

This is an equation for the four components of the metric tensor of a curved spacetime, in which, $R_{\mu v}$ is the Ricci curvature tensor, $R$ is the scalar curvature, $g_{\mu v}$ is the metric tensor of the space-time geometry, $G$ is the gravitational constant, $T_{\mu \nu}$ is the stress-energy tensor of the matter universe and $\Lambda$ is the well known cosmological constant with dimension of (length) $)^{-2}$. However, when Hubble discovered that the universe effectively is expanding, Einstein considered the inclusion of the cosmological term as his biggest blunder.

In the Friedmann-Lemaitre-Robertson-Walker universe, the effect of the local gravitational sources can be seen as local perturbations. Such a universe usually is modeled in terms of the four-dimensional energy-momentum tensor of a homogeneous and isotropic perfect fluid (uncorrelated particles), with a spatially constant vacuum energy density $\rho_{v}$ and an isotropic negative pressure $p$ :

$$
T_{\mu v}=\left(\rho_{v}+p\right) U_{\mu} U_{v}+p g_{\mu v}
$$

where $U_{\mu}\left(U_{v}\right)$ is the local four-velocity of the perfect fluid and $g_{\mu, v}$ is the metric tensor.

In the absence of ordinary matter-energy (empty and static universe), $T_{\mu v}$ in Equation (2) reduces to $T_{00}$, which is interpreted as the energy density of the vacuum $\rho_{v}$. From the perspective of the elementary particle physics, the cosmological constant is proportional to the energy density of the vacuum and, importantly, the local density of this vacuum energy remains constant during the expansion of the universe [6] [7]. Therefore, expansion creates additional vacuum energy, which leads to the odd negative pressure of the perfect fluid. The vacuum of elementary particle physics usually includes the zero-point energies, associated with the various force fields. Altogether, these contributions lead to the theoretical vacuum energy density $\rho_{v}^{\text {th }}$, given by:

$$
\rho_{v}^{\text {th }}=10^{110} \mathrm{erg} / \mathrm{cm}^{3} .
$$

However, the experimental observations, with the help of $I_{a}$ supernovae, [8] [9] as well as with the help of cosmic microwave background radiation, [10] showed that the universe is not only expanding. The expansion rate is accelerat- 
ing. The big question then is: What causes the accelerating expansion of the universe? These experimental observations also have provided approximate values for the actual vacuum energy density $\rho_{v}^{o b s}$ given by:

$$
\rho_{v}^{o b s} \sim 10^{-10} \mathrm{erg} / \mathrm{cm}^{3}
$$

The gap between the theoretical estimation in Equation (3) and the experimental observations in Equation (4) amounts to the scandalous 120 decimal orders of magnitude [6] [7] and decreases not much, even with the most favorable estimates. This enormous difference has revived Einstein's dilemma about the cosmological constant. It lets clear that something very fundamental is wrong with the assumption of particle physics about the nature of empty space (vacuum). The Robertson-Walker universe cannot be described in terms of a perfect fluid and the vacuum energy is not the zero-point energy of the ground states of the various force fields. This makes the discussion about the vacuum energy density and the cosmological constant more actual than ever.

The accelerating expansion of the universe is actually a well established observational fact. The consensus among the cosmologists is that dark energy is not ordinary mass-energy, however is energy of empty space (vacuum) itself. It also is consensus that the energy of the vacuum distributes it very homogeneously throughout the universe. The goal of the present work is showing that all these features arise naturally and appropriately within the context of the global Higgs Quantum Space (HQS) dynamics.

It is totally clear that within the current view about the nature of empty space, a solution of the vacuum energy density problem is well out of reach. Only radical changes can open the way to a solution. The present work challenges this impasse within the new scenario of the Higgs theory [11] [12]. Recent experimental observations in the LHC in Geneva $\mathrm{CH}$ give support to the Higgs theory. According to this theory, empty space (vacuum) is not empty at all. It is filled up by a quantum fluid medium, a quantum condensate or Higgs condensate (HC) that is analogous to the superconducting condensate (SCC). However, while the condensation of the SCC occurs only at very low temperatures and is stabilized by an energy gap of only $1 \mathrm{meV}$, the $\mathrm{HC}$ is believed to have initiated condensation at $10^{15} \mathrm{~K}$ and is stabilized by a huge energy gap that, according to the Glashow-Weinberg-Salam electroweak model, achieves more than $200 \mathrm{GeV}$.

The current theories describe empty space in terms of a perfect fluid and the zero-point energy of a huge (almost infinite) number of uncorrelated oscillators. However, quantum condensates and in particular the HC, far from a perfect fluid of uncorrelated particles, are strongly correlated and highly phase coherent macroscopic quantum states of bosons with broken $U(1)$ symmetry and ruled by a very strong order parameter (collective wave-function). While in perfect fluids the particles respond individually to a perturbing field, in quantum condensates, the response always is collective involving all the particles of the condensate, through their order parameter. This turns the quantum condensate able to develop a screening velocity field, confining the perturbing field by effects like 
the well-known Meissner effect in superconductors. This is believed to take place too in the HQS and is known as Higgs mechanism. Quantum condensates are like an army company, in which any attack always is an attack against all the members and has the coordinated response of the entire company.

The huge energy gap of the HC or Higgs Quantum Space (HQS) turns its order parameter extremely stable and rigid. It behaves like one unique entity (one unique oscillator) the size of the universe. Due to this huge energy gap, the order parameter of the HQS very strongly suppresses local quantum fluctuations and the zero-point energies. Therefore, the usual estimates of the vacuum energy density, in terms of quantum fluctuations and the zero-point energies of a perfect fluid, cannot be correct. On the other hand, local steady state excitations (local Goldstone modes) of the HQS require high energies, in the order of MeVs. However, the HQS at the same time is perfectly conservative. Therefore, excitations once created, automatically become indefinitely persistent.

The vacuum fluctuations causing the Casimir effect and the Lamb shift in Quantum Electrodynamics are often claimed to corroborate the correctness of the methods of particle physics to estimate the vacuum energy density. However, both these effects are of electromagnetic (EM) nature. The EM field has its $U(1)$ symmetry preserved and is long-range. It is not a quantum fluid. Moreover, the contribution of the zero-point fluctuations of the EM field to the vacuum energy density is very low. Contrarily, the Higgs Quantum Space (HQS) is an integrated entity, ruled by a very strong and rigid order parameter, which means wave lengths of the Higgs modes tending to the infinite and to zero frequency.

The next Section 2 outlines with more details the nature and physical properties of the HQS filling up the empty space. As the properties of the HQS are closely analogous to the well-known properties of the superfluids and the superconducting condensates, the well known properties of these quantum fluids can be used as paradigm. Many of these properties are generic to all quantum condensates. The coming Sections 3 and 4 will discuss the origin of the accelerating expansion of the universe from the perspective of the theoretical and experimental knowledge respectively, showing that within the scenario of the HQS, the vacuum energy density naturally approaches the observed value.

\section{The Nature of the Empty Space}

According to the Higgs theory, immediately after the big-bang, when the temperature fell through $10^{15} \mathrm{~K}$ a scalar field, present throughout space, has caused the breakdown of the electroweak symmetry into the weak force doublet $S U(2)$ and the electromagnetic (EM) field. While the EM field remained with its $U(1)$ symmetry unbroken, the weak force doublet has spontaneously broken its $U(1)$ symmetry and given rise to the Higgs condensate (HC). The HC is stabilized by a huge energy gap, which, according to the Glashow-Weinberg-Salam electroweak model, achieves more than $200 \mathrm{GeV}$ 
[6] [7]. The condensation energy of the HC plays the role of the vacuum energy and of the cosmological constant in Equation (1). It will be seen to be ultimately responsible for the accelerated expansion of the universe. The present work will not discuss details of the Higgs theory; however will rather implement its important practical consequences and the role of the HQS in the life of the universe.

Bose-Einstein (BE) phase correlation between the wave functions of the bosons enforces phase coherence and breakdown of the $U(1)$ symmetry, because this lowers the energy of the boson system. In this phase transition all the bosons condense into the same quantum ground state, in which they all assume the same phase, thereby forming a highly coherent quantum fluid state. Their wave-functions entangle, turning the bosons indistinguishable. This condensation is a second order phase transition that involves no latent heat. The condensation energy is released gradually down to absolute zero temperature. The Higgs condensate is a quantum fluid medium, ruled by the principles of quantum physics and with properties closely analogous to the superconducting condensate. It fills up the whole of space and is the quintessence of the empty space (vacuum). Likewise the conventional quantum condensates, the HQS too is intrinsically highly homogeneous throughout the universe.

Analogously as the superconducting condensate (SCC), the Higgs condensate too can be described by a Ginsburg-Landau like [13] complex order parameter $\Phi(r, \theta)=\phi(r) \mathrm{e}^{i \theta}$, a collective wavefunction in which $\phi(r)$ is the amplitude and $\mathrm{e}^{i \theta}$ is the phase factor. $\Phi\left(r, \theta_{0}\right)=\phi(r) \mathrm{e}^{i \theta_{0}}$ is the resting condition, the ground state of the condensate and $\rho=\Phi^{*} \Phi$ is the local condensate density that intrinsically is constant throughout the volume of the condensate.

$\mathrm{BE}$ phase correlation between the boson wefunctins gives rise to a negative potential energy (bonding) term, the value of which increases linearly with the condensate density $\rho$. Another positive potential energy (anti-bonding) term arises from repulsive core interaction between the bosons that increase with the squared density $\rho^{2}$ and prevents collapse of the system. The effective potential is:

$$
V(\rho)=-n \rho+m \rho^{2}
$$

where however the negative coefficient $(-n)$ of the bonding term is considerably larger than the positive coefficient $(m)$ of the anti-bonding term. Because of the negative coeficient $(-n)$, the minimum of the effective potential occurs not for $\rho=0$, however for a finite value $\rho=n / 2 m$. This is known as a non-zero vacuum-expectation-value that is homogeneous throughout the volume of the condensate. In terms of the components of $\Phi$ the Higgs potential energy well has the form of a Mexican sombrero. Figure 1 depicts the form of this potential well in terms of the Real and Imaginary components of the order parameter. This form of the potential is characteristic of all quantum condensates.

The deepness of the potential well depends on the strength of the phase correlation between the bosons as well as on the phase coherence length. While in the conventional quantum fluids (superfluids and SCC) the coherence length is 


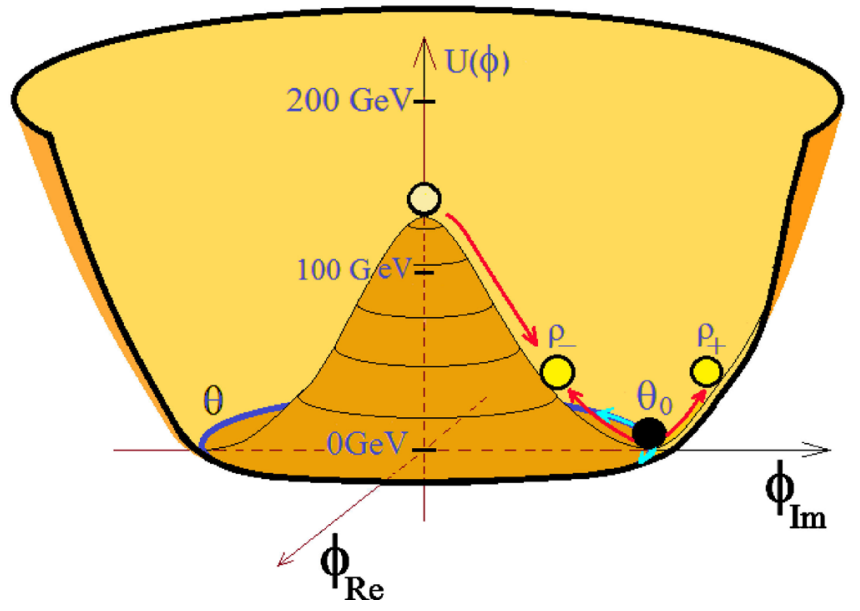

Figure 1. Characteristic potential well created during Bose-Einstein Condensation. The figure depicts locally the form of the Mexican sombrero potential in terms of the Real and the Imaginary components of the order parameter, where the given energy scale is for the Higgs condensate. A red arrow indicates the transition toward the lower energy phase coherent state with the well-defined phase $\theta_{0}$. The figure also indicates the low volumetric density $\left(\rho_{-}\right)$and the high volumetric density $\left(\rho_{+}\right)$situations. While $\rho_{-}$drives accelerating contraction, $\rho_{+}$drives accelerating expansion of the condensate. This is related with the Higgs mode. The global Goldstone mode is indicated along the blue bottom circle.

rather short (tens of Angströms) and the deepness of the potential well is of only $1 \mathrm{meV}$, in the Higgs condensate it achieves hundreds of $\mathrm{GeV}$ and its phase coherence may be very long-range. The fact that the phase of the condensate $\left(\theta_{0}\right)$ can take any value between 0 and $2 \pi$, without changing the energy, proves that the spontaneous breakdown of the $U(1)$ symmetry preserves the gauge symmetry of the Lagrangian.

The Higgs theory introduces profound changes in Einstein's view about the nature of empty space (vacuum). Being responsible for the inertial mass, the HQS is not only a local ultimate reference for rest and for motions of matter-energy, however literally governs these motions. The HQS, giving mass to the elementary particles, too is responsible for the gravitational dynamics; because it is mass that creates the gravitatonal fields. The Higgs theory provides by the first time a scientifically sound explanation for the origin of inertial mass of the elementary particles. The origin of the inertial mass is intimately related with the persistence of all excitations in the HQS. The Higgs theory pictures to us a universe in which the HQS plays a fundamental role both in the microscopic world of quantum physics as well as in the macroscopic world of gravitation. The HQS thus links together the physics of the quantum world and the physics of gravitation, which opens the way for their unification [14].

The nature of the HQS is profoundly different from that a perfect fluid. In a perfect fluid the particles are all independent, well localized and have their $U(1)$ symmetry preserved. In such a perfect fluid the particles are uncorrelated and respond individually to any perturbing field. This turns this perfect fluid 
unable to develop collective and cooperative effects and is why these perfect fluids cannot confine, quantize an interacting field, nor can it develop a mechanism creating inertial mass for it. Contrarily, quantum fluids are formed by strongly correlated bosons with spontaneously broken $U(1)$ symmetry and condensed into a unique long-range phase coherent macroscopic quantum state. Such a quantum fluid is an integrated system, ruled by a complex order parameter. The HQS is governed by an extremely strong and powerful order parameter, which means that Higgs modes have extremely long wavelength tending to infinity. Only high energies can excite local (Goldstone like) modes with shorter wavelengths. They however automatically become persistent and are not virtual particles, because the HQS strongly suppresses quantum fluctuations and is perfectly conservative. The amplitude of the Higgs order parameter is highly uniform throughout the volume of the universe. This can solve the horizon and the flatness problems.

\section{Nature of the Energy Accelerating the Expansion of the Universe}

On spontaneously breaking down the $U(1)$ symmetry, the spin zero Higgs particles create a deep potential energy well of the form depicted in Figure 1. They condense into a negative energy state liberating the corresponding energy difference. In this process the total energy is conserved. Nothing is created from nothing. As Stephen Hawking liked to say, in order to create a mountain, it is enough to excavate a big hole. If the universe is an adiabatic system the energy liberated during the condensation remains within the universe in various forms. Such a behavior is well-known from the condensation of the usual ${ }^{4} \mathrm{He}$ into a superfluid, in which the condensation energy can in part be converted into quasi particles (Rotons, Maxons and vortices). In the condensation of superfluids and superconducting condensates a considerable amount of heat energy is liberated, which normally is removed by efficient cryogenics. Insufficient removal or dissipation of the condensation energy necessarily slows down or even stops the condensation rate.

The Higgs condensate (HC) fills up the space of the whole universe. To now, nothing indicates that an external world exists that can remove and absorb the very huge amount of condensation energy. Therefore, the only possible way to the universe reducing its energy density and temperature is by adiabatic expansion, converting its energy into kinetic energy of expansion and into confined mass-energy particles. The condensation of the Higgs condensate necessarily is an adiabatic process, analogous to the condensation of clouds, during the ascension and adiabatic expansion of warm and humid air. In the Higgs boson condensation however no external pressure opposes the expansion. Therefore, the expansion rate is free and accelerated. In this adiabatic process, the total energy must be conserved. The only way to lower the energy density and the temperature of the $\mathrm{HC}$ and of the universe is by volumetric expansion. Along this 
process, a large part of the condensation energy is converted into mass-energy and kinetic energy of the expanding universe. This expansion stretches the wavelengths of the particles and of radiation as a function of time, thereby reducing the momentum and kinetic energy of the particles, according to the de-Broglie equation $(p=h / \lambda)$ with respect to the local HQS. This is why actually all matter in the universe is very closely stationary with respect to the local moving HQS and the temperature is close to zero. It also lowers the frequency and the energy of the radiation from distant sources (Hubble red-shift) and has shifted the frequency of the primordial Hydrogen radiation to the microwave frequency.

The presence of the ordinary fermion matter (concentrated mainly in stars and galaxies) represents a source of persistent phase perturbation and phase disorder of the Higgs order parameter. This holds back the advance toward the fully broken $U(1)$ symmetry and to the minimum of energy in the $\mathrm{HC}$ in the potential well Figure 1. The actual low temperature of the universe, of about $2.7 \mathrm{~K}$, indicates that the universe lies deeply, near to the bottom of the Higgs potential well.

From the perspective of the present work, the total energy $E$ of the universe may be calculated by the equation:

$$
\begin{aligned}
E & =\int_{V(t)} \rho_{v}(t) \mathrm{d} V=\rho_{v}(t) \int_{V(t)} \mathrm{d} V \\
& =\rho_{v}(t) V(t)=E_{0}=\text { Constant }
\end{aligned}
$$

In this Equation, $V(t)$ is the total volume of the universe as a function of time, $\rho_{v}(t)$ is the time dependent vacuum energy density that is highly uniform (constant) over the volume of the universe and $E_{0}=200 \mathrm{GeV} \times V_{0}$ is the total initial energy of the universe with respect to the bottom of the potential well at the epoch of the electroweak break-down, which must be conserved.

The energy of the HC within the Higgs potential well depends on the volumetric density $\rho=\Phi^{*} \Phi=|\phi|^{2}$. If $\rho>n / 2 m$ or $\rho<n / 2 m$, the energy of the HC is not minimum. Please see Figure 1. If $\rho>n / 2 m$ and the number of Higgs particles is fixed, the universe can lower its energy by accelerated expansion. The Higgs condensate (HC) and with it the matter-energy universe lie deeply within the Higgs potential energy well Figure 1, however not at the minimum of energy. The matter fields, perturbing the order parameter and causing phase disorder of the Higgs order parameter, hold back the advance toward the minimum of energy. The situation is analogous to that of a superconductor below the transition temperature, however under a strong applied magnetic field. The phase perturbations of the magnetic field on the superconducting order parameter too hold back the advance of the SCC toward its minimum of energy. It can even destroy superconductivity for sufficiently high fields. Observations show that actually the expansion rate of the universe is accelerating. This indicates that $\rho>n / 2 m$. However, if $\rho<n / 2 m$, the HC can lower its energy by accelerated contraction. Apparently the universe can cycle between periods of free expansion and of free contraction. 
Due to the very strong suppression of the fluctuations and of the zero-point energies of the various force fields by the HQS, all these contributions to the vacuum energy density are negligibly low. The only relevant vacuum energy is the condensation energy liberated during breakdown of the $U(1)$ symmetry. However, to parameterize the vacuum energy density as a function of the volume of the universe is difficult, because the size of the universe at the epoch of the electroweak symmetry and the $U(1)$ symmetry breakdowns is not well known. Moreover, this condensation energy was liberated only gradually as the temperature fell. The temperature of the universe is a better parameter. The electroweak symmetry break-down is believed to have initiated closely after the big-bang, when the temperature fell through temperatures between $10^{15} \mathrm{~K}$ and $10^{12} \mathrm{~K}$. On from the recombination epoch and formation of the Hydrogen atoms, the temperature gradually fell down to the actual $2.7 \mathrm{~K}$, known from the cosmic-microwave-background radiation. However, the average temperature of the universe certainly is a little bit higher, because of the hot stars and galaxies. Maybe $3 \mathrm{~K}$ is a good guess.

$$
\rho_{v}=200 \mathrm{GeV} \times \frac{3}{10^{12}}=0.6 \mathrm{eV} \sim 2 \times 10^{-10} \mathrm{ergs} / \mathrm{cm}^{3}
$$

This result is equivalent to about $10^{-29} \mathrm{~g} / \mathrm{cm}^{3}$ (about 6 Hydrogen atoms per cubic meter). However, although this mass-energy density is very low, it is constant over the entire space of the universe. Integrating it, gives a value about 14 times that of the visible mass-energy in the universe. This last however is confined in the astronomical bodies. On the other hand, the well known relation between the vacuum energy density $\rho_{v}$ and the cosmological constant $\Lambda$ is:

$$
\Lambda=\frac{8 \pi G \rho_{v}}{c^{2}} \sim 10^{-52} \mathrm{~m}^{-2}
$$

where $G$ is the gravitational constant and $c$ is the velocity of light in the vacuum.

The present calculation of the vacuum energy density, while giving values close to the observations, also helps to understand the intriguing coincidence problem of cosmology. Why are actually the values of the vacuum energy and of the visible ordinary matter-energy so closely similar? The actual small residual vacuum energy density consists of two parts: The condensation energy that still has not decayed because the temperature of the universe is not absolute zero (bare cosmological constant). According to the present work, the phase perturbation of the Higgs order parameter by the ordinary (visible) matter-energy in the universe is responsible for the major part of the residual vacuum energy density. This explains why actually the total vacuum energy and the total visible matter energy are so closely similar. On the other hand, the only relationship of the HQS, with ordinary matter-energy, is giving mass to the elementary particles and ruling their inertial motion. In this scenario, the only way of the HQS-dynamics (vacuum energy) to affect the motion of ordinary matter-energy is by inertial dynamics effects, which after Einstein's principle of equivalence are gravitational effects. 


\section{Experimental Evidence That the Accelerating Expansion of the Universe Keeps to the Accelerating Expansion of the HQS Itself}

Clocks, stationary within gravitational fields, are well known to show exactly the gravitational slowing predicted by GR. However, the GPS clocks moving with earth round the sun show no sign of the gravitational slowing by the solar field [15] [16] predicted by the GR. This observation, together with the null results of the Michelson light anisotropy experiments, demonstrates that the HQS, ruling the motion of matter-energy, is circulating round the sun according to a Keplerian velocity field, consistently with the planetary motions. It certainly is also moving round every other astronomical body consistently with the local main astronomical motions and generating the respective gravitational fields. The HQS materializes the local Lorentz Frames (LFs) turning them into local proper LFs. This Keplerian velocity field of the HQS is the quintessence of the gravitational fields and is shown in Refs. [14] [17] to accurately create the observed gravitational dynamics on earth, in the solar system and also to create the observed non-Keplerian rotation of the galaxies without the need of dark matter. It moreover produces correctly all the observed effects of the gravitational fields on light and on clocks. In the Keplerian velocity field of the sun, earth and all the other planets of the solar system are closely stationary with respect to the local moving HQS and thus with respect to the local proper LFs. The planets simply are carried round the sun by the solar Keplerian velocity field of the HQS. The solar system too is stationary with respect to the local HQS in the galactic velocity field and carried around the center of the Milky-Way gralaxy. The observed absence of the gravitational slowing of the GPS clocks by the solar field and the isotropy of light with respect to earth are both obvious signatures of the physical mechanism of gravity in action in the solar field.

The Keplerian velocity field of the HQS round the sun and the velocity field round the galactic center properly explain the isotropy of light with respect to earth. It however cannot explain why the recession between the galaxies too causes no light anisotropy. The fact that the velocity of light is isotropic with respect to earth, despite its orbital motion round the sun and despite the orbital motion of the solar system in the Milky-Way galaxy and also despite the relative motion and recession between the galaxies, demonstrates that the accelerated recession between the galaxies too lets earth stationary with respect to the local moving HQS. Despite the accelerated expansion of the universe, our Milky-Way galaxy remains stationary with respect to the local HQS. It certainly would not be reasonable to assume that our galaxy is in a privileged kinematic circumstance with respect to the HQS in detriment to all the other galaxies in the universe. All the galaxies must equally be closely stationary with respect to the local moving HQS. These facts entail the conclusion that the HQS, governing the inertial motion of matter and propagating light, besides moving round earth, round the sun and round the center of the Milky-Way galaxy, according to ve- 
locity fields, consistent with the local main astronomical motions also is expanding consistently with the recession between the galaxies.

Within this scenario, the Hubble spectral red-shifts of light from distant galaxies are not usual Doppler shifts and the red-shift of the cosmic microwave background radiation are due to the time-rate of stretching of the wavelength, due to the expansion of the HQS, their medium of propagation and the ultimate reference for rest and for motions. It is easy to show that this stretching of the wavelength, as a function of time, causes exactly the same red-shift as the usual Doppler shift, due to conventional recession. The expansion of the HQS, stretching the wavelength of the particles and of light, reduces the kinetic energy of the particles with respect to the local HQS and the energy of the radiation. Moreover, the velocity of oppositely moving matter bodies is averaged down and converted into heat during the gravitational agglomeration into the large astronomical bodies. These observations naturally explain why the velocity of all the large astronomical bodies with respect to the local HQS is actually so small (only hundreds of meters per second) throughout the universe.

In summary, the HQS, ruling the inertial motion of matter and the propagation of light, is circulating round earth, round the sun and round the galactic center, consistently with the local main astronomical motions. These velocity fields are the quintessence of the gravitational fields. In the local HQS-dynamics round the sun and round the galactic center, earth is very closely stationary with respect to the local moving HQS, so that the orbital motion of earth and the motion of the solar system within the galaxy cause no relevant light anisotropy with respect to earth. The observed absence of the gravitational slowing of the GPS clocks by the solar field perfectly corroborates this conclusion. The null light anisotropy results however demonstrate in addition that the accelerating expansion of the universe too lets earth, the solar system and the Milky-Way galaxy and all the other galaxies stationary with respect to the local HQS. This concomitant expansion of the HQS and of the matter universe ultimately explains the observed isotropy of light with respect to earth and shows that the accelerated expansion of the visible universe keeps to the accelerating expansion of the HQS itself. The fact that all the astronomical bodies throughout the universe are very closely stationary with respect to the local HQS predicts the universality of the laws of physics.

Dark energy is not ordinary mass-energy, but is energy of the HQS itself, energy of the vacuum. The total vacuum energy is closely similar to the total visible matter-energy, because it is the visible matter-energy that holds back the HC from its minimum of energy. Also, due to the fact that the HQS is a quantum fluid, ruled by an order parameter, this energy necessarily distributes it very homogeneously throughout the universe. Moreover, the HQS, ruling the inertial motion of matter-energy, interacts with ordinary matter-energy only by inertial effects, causing inertial dynamics, which, according to Einstein's Equivalence Principle, are gravitational effects. This shows that the HQS-dynamics naturally 
meets all the characteristic properties of dark energy that are consensual among the cosmologists. It however entails a fundamental additional feature: The accelerating expansion of the universe perfectly keeps to the accelerated expansion of the HQS itself.

\section{Conclusion}

The Higgs Quantum Space (HQS) is a very strongly correlated quantum fluid medium, ruled by an order parameter and stabilized by a huge energy gap. This order parameter very strongly suppresses the local quantum fluctuations and the zero-point energies of the weak and strong nuclear fields, turning their contribution to the vacuum energy density irrelevant. Therefore, the estimate of the vacuum energy density, in terms of the zero-point energies leads to a completely wrong value. On the other hand, the universe is an adiabatic system. The only possible way of it reducing its energy density and temperature is by expansion, converting its energy partly into kinetic energy of expansion and partly into confined mass-energy. The fact that the actual residual energy is mostly due to the phase disorder, created by the presence of the ordinary mass-energy, explains the actual similarity of the total vacuum energy and of the totally visible matter-energy. Finally, the zero light anisotropy on earth demonstrates that, in the accelerated expansion of the universe, the visible matter universe keeps to the accelerated expansion of the HQS itself.

\section{Conflicts of Interest}

The author declares no conflicts of interest regarding the publication of this paper.

\section{References}

[1] Friedmann, A. (1924) Zeitschrift für Physik, 21, 326-332. https://doi.org/10.1007/BF01328280

[2] Einstein, A. (1916) Annalen der Physik, 49, 769-822.

[3] Lorentz, H.A., Einstein, A., Minkowski, H. and Weyl, H. (1923) The Principle of Relativity. Dover Publications, New York.

[4] Robertson, H.P. (1936) Astrophysical Journal, 83, 257. https://doi.org/10.1086/143726

[5] Walker, A.G. (1937) Proceedings of the London Mathematical Society, 42, 90-127. https://doi.org/10.1112/plms/s2-42.1.90

[6] Carrol, S.M. (2000) Determination of Dark Energy and Dark Matter from the Values of Redshift for the Present Time, Planck and Trans-Planck Epochs of the Big-Bang Model. arXiv:astro-ph/0004075v

[7] Sola, J. (2013) Cosmological Constant and Vacuum Energy: Old and New Ideas. arXiv:1306.1527v3 [gr-qc]

[8] Riess, A., et al. (1998) The Astronomical Journal, 116, 1009. https://doi.org/10.1086/300499

[9] Perlmutter, S., et al. (1999) The Astronomical Journal, 517, 565. https://doi.org/10.1086/307221 
[10] Lobo, F.S.N. (2006) Classical and Quantum Gravity, 23, 1525. https://doi.org/10.1088/0264-9381/23/5/006

[11] Higgs, P.W. (1964) Physical Review Letters, 13, 508. https://doi.org/10.1103/PhysRevLett.13.508

[12] Englert, F. and Brout, R. (1964) Physical Review Letters, 13, 321. https://doi.org/10.1103/PhysRevLett.13.321

[13] Ginzburg, L. and Landau, L.D. (1950) Journal of Experimental and Theoretical Physics (JETP), 20, 1064.

[14] Schaf, J. (2018) Journal of Modern Physics, 9, 395. https://doi.org/10.4236/jmp.2018.93028

[15] Hatch, R.R. (2004) GPS Solutions, 8, 67-73. https://doi.org/10.1007/s10291-004-0092-8

[16] Hatch, R.R. (2004) Foundations of Physics, 34, 1725-1739. https://doi.org/10.1007/s10701-004-1313-2

[17] Schaf, J. (2018) Journal of Modern Physics, 9, 111. https://doi.org/10.4236/jmp.2018.95068 